Gut, 1988, 29, 1539-1543

\title{
Short chain fatty acid distributions of enema samples from a sigmoidoscopy population: an association of high acetate and low butyrate ratios with adenomatous polyps and colon cancer
}

\author{
G A WEAVER, J A KRAUSE, T L MILlER, AND M J WOLIN
}

From the Department of Medicine, The Mary Imogene Bassett Hospital (affiliated with Columbia University), Cooperstown, New York and Wadsworth Center for Laboratories and Research, New York State Department of Health, Albany, New York, USA

SUMMARY We investigated the distribution of short chain fatty acids (SCFA) in enema samples taken from subjects before sigmoidoscopy as an indicator of possible microbial community differences between subjects subsequently diagnosed as normal or having colonic disorders. The major SCFA in all groups were acetic, propionic, and butyric acids. A significantly higher ratio of acetate to total SCFA and lower ratio of butyrate to total SCFA was found for polyp-colon cancer subjects than for normal subjects. There were no significant differences in the ratios of acetate, propionate, or butyrate between the diverticulosis or inflammatory bowel groups and the normal group. There were no significant sex differences nor were there correlations with the ratios of acetate, propionate or butyrate and age, subject weight, or dry weights of samples. Significant differences in concentrations of individual acids were found between normal and certain diagnostic groups. The difference in proportions of individual SCFA between groups suggest differences in fermentation patterns of the colonic microflora.

The major products of bacterial fermentation in the colon are short chain fatty acids (SCFA), $\mathrm{CH}_{4}, \mathrm{H}_{2}$, and $\mathrm{CO}_{2} \cdot{ }^{1}$ The overall fermentation and the final products are the result of interactions between the different species of anaerobic bacteria of the colon.' A factor that may cause variations in the species composition of the microbial community and the resulting fermentation is the substrate for the overall fermentation which may vary in vivo because of differences in diet or differences in absorption. We recently showed that there were consistent differences between two individuals over a three and a half year study period in the ability of their microbial communities to ferment starch, the distributions of SCFA formed from glucose or starch and in the distributions of SCFA in faecal specimens. ${ }^{2}$ Production of methane may be less dependent on the

Address for correspondence: G A Weaver, The Mary Imogene Bassett Hospital, Cooperstown, New York 13326, USA.

Received for publication 5 May 1988. substrate available for fermentation in the colon. The amounts of colonic methane produced and the concentration of the responsible species of methane producing bacteria vary significantly between individuals but are relatively constant within individuals. ${ }^{2-6}$

Subjects with colonic disease might have colonic microbial communities and fermentations that differ from normal. Therefore we investigated the distribution of the major SCFA (acetate, propionate, and butyrate) and minor SCFA (isobutyrate, isovalerate, and valerate) in enema samples taken from subjects before sigmoidoscopy as an indicator of possible microbial community differences between subjects subsequently diagnosed as normal or with other colonic disorders. The results presented in this report show significant differences in the concentrations and proportions of the major SCFA in subjects with colon polyps or colon cancer as compared with normal subjects. The major SCFA of subjects with 
inflammatory bowel disease or diverticulosis did not differ significantly from normal subjects except for a higher concentration of propionate in the diverticulosis groups.

\section{Methods}

\section{POPULATION}

The subjects from a sigmoidoscopy population were previously described in a report of a study of the incidence of methanogenic bacteria in human faecal flora. ${ }^{5}$ During that study, portions of tap water enema samples were frozen at the time of collection, as described below, for later analysis of SCFA concentrations. Of the 130 samples examined for methanogenic bacteria, 115 were available for SCFA analysis. Subjects were placed in diagnostic groups as previously described. ${ }^{5}$ Subjects were placed in the following groups for statistical analysis. Some of the groups overlap. (Mean and standard deviation (SD) of ages are in square parentheses):

NORMAL

Thirty five (54 [12.5]) no subjects with colon abnormality.

POLYPS AND/OR COLON CANCER (NO

DIVERTICULA)

Eighteen (62.5 [13.9]). No subjects with diverticulosis are in this group. Two subjects had colon cancer without evidence of polyps, thus the designation polyps and/or colon cancer.

POLYPS AND/OR COLON CANCER (INCLUDING DIVERTICULA)

Thirty three $(63.8[13.0])$. This group includes all subjects with polyps and/or cancer. Fifteen of these subjects had the presence of polyps and/or colon cancer and diverticulosis.
DIVERTICULA (NO POLYPS)

Thirty nine $(62.9$ [13.0]). No subjects with polyps and/or colon cancer are included in this group.

\section{DIVERTICULA (INCLUDING POLYPS AND/OR} COLON CANCER)

Fifty four (63.6 [12.7]). All subjects with diverticulosis are included in this group. This group also includes the 15 subjects with diverticulosis and/or colon cancer.

COLON CANCER (INCLUDING DIVERTICULA) Eleven $(67 \cdot 7$ [7.5]). All subjects with colon cancer including some with diverticulosis.

\section{INFLAMMATORY BOWEL DISEASE}

Eight $(41.6[19 \cdot 3])$. All subjects with inflammatory bowel disease.

This study was initially reviewed and approved by the review board for human subjects of The Mary Imogene Bassett Hospital in July, 1981, with subsequent yearly approvals.

\section{SAMPLE PREPARATION}

Portions of tap water enema suspensions obtained from the sigmoidoscopy population ${ }^{5}$ were transferred to serum bottles while flushing the headspace of the bottles with $100 \% \mathrm{~N}_{2}$. The bottles were anaerobically sealed and stored at $-20^{\circ} \mathrm{C}$. Before SCFA analysis, a sample was rapidly thawed under warm running water and centrifuged for 10 minutes at $20000 \times g$. The supernatant portion of each sample was used to determine SCFA concentrations as described below.

DRY WEIGHT DETERMINATIONS

The gram dry weight (gdw) of duplicate portions of enema samples $(5-15 \mathrm{ml})$ was determined as previously described. ${ }^{5}$

Table 1 Faecal SCFA to total SCFA ratios in normals and subjects with adenomatous polyps and/or diverticulosis

\begin{tabular}{|c|c|c|c|c|c|c|c|}
\hline \multirow[b]{2}{*}{ Diagnostic category } & \multicolumn{7}{|c|}{$\%$ molar ratios* } \\
\hline & $n$ & Acetate & Propionate & Isobutyrate & Butyrate & Isovalerate & Valerate \\
\hline Normal & 35 & $60 \cdot 7(8 \cdot 7)$ & $15 \cdot 8(5 \cdot 2)$ & $1 \cdot 7(1 \cdot 3)$ & $17 \cdot 3(6 \cdot 7)$ & $2 \cdot 2(1 \cdot 4)$ & $2.4(1.0)$ \\
\hline \multicolumn{8}{|l|}{ Polyp-cancer } \\
\hline No diverticula & 18 & $69 \cdot 2(6 \cdot 8)[0 \cdot 001]$ & $14 \cdot 8(5 \cdot 4)$ & $1 \cdot 1(0 \cdot 5)$ & $11 \cdot 5(3.6)[0.0015]$ & $1 \cdot 5(0 \cdot 7)$ & $2 \cdot 0(1 \cdot 3)$ \\
\hline Including diverticula & 33 & $67 \cdot 1(7 \cdot 1)[0 \cdot 0021]$ & $15 \cdot 5(4 \cdot 9)$ & $1 \cdot 1(0 \cdot 6)[0 \cdot 033]$ & $12 \cdot 3(3 \cdot 6)[0 \cdot 0006]$ & $1 \cdot 8(0 \cdot 8)$ & $2 \cdot 2(1 \cdot 4)$ \\
\hline Colon cancer & 11 & $67 \cdot 3(5 \cdot 9)[0 \cdot 0246]$ & $16 \cdot 8(4 \cdot 9)$ & $1 \cdot 1(0 \cdot 5)$ & $11 \cdot 2(4 \cdot 0)[0 \cdot 0065]$ & $1 \cdot 5(0 \cdot 6)$ & $2 \cdot 1(1 \cdot 5)$ \\
\hline \multicolumn{8}{|l|}{ Diverticula } \\
\hline No polyp-cancer & 39 & $61 \cdot 8(8.9)$ & $16 \cdot 0(4 \cdot 4)$ & $1 \cdot 6(1 \cdot 5)$ & $16 \cdot 0(6 \cdot 2)$ & $2 \cdot 1(1 \cdot 5)$ & $2 \cdot 5(1 \cdot 4)$ \\
\hline Including polyp-cancer & 54 & $62 \cdot 3(8 \cdot 4)$ & $16 \cdot 1(4 \cdot 3)$ & $1 \cdot 5(1 \cdot 3)$ & $15 \cdot 3(5 \cdot 7)$ & $2 \cdot 1(1 \cdot 3)$ & $2 \cdot 5(1 \cdot 4)$ \\
\hline Inflammatory bowel disease & 8 & $67 \cdot 4(8 \cdot 1)$ & $15 \cdot 3(6 \cdot 8)$ & $1 \cdot 0(0 \cdot 8)$ & $12 \cdot 9(3 \cdot 3)$ & $1 \cdot 4(1 \cdot 2)$ & $2.0(1.9)$ \\
\hline
\end{tabular}

*Means (SD); p values comparing the means of diagnostic groups with the means of the normal group by a two tailed $t$ test are shown in square brackets where significant. 
SHORT CHAIN FATTY ACID ANALYSIS

Samples were analysed using a Perkin Elmer gas chromatograph with a flame ionization detector and $1.82 \mathrm{~m} \times 0.63 \mathrm{~cm}$ glass column packed with $15 \% \mathrm{SP}$ $1220 / 1 \% \mathrm{H}_{3} \mathrm{PO}_{4}$ on 100/120 Chromosorb W AW (Supelco, Inc, Supelco Park, Bellefonte, PA 168230048). Short chain fatty acids were ether extracted as described previously ${ }^{2}$ and a $1 \mu$ l sample was used for chromatographic injection. Short chain fatty acid concentrations were determined with a HewlettPackard 3390A Integrator by the internal standard method. Per cent recovery from SCFA spiked samples for each SCFA was: acetate 108\%, propionate $99 \%$, isobutyrate $96 \%$, butyrate $100 \%$, isovalerate $90 \%$, and valerate $92 \%$. Coefficients of variation for repeated determinations were less than $6 \%$.

\section{Results}

The major SCFA in all groups were acetate, propionate, and butyrate (106 to $1146 \mu \mathrm{mol} / \mathrm{gdw}$ ) and valerate, isovalerate, and isobutyrate were present as minor components (9-24 $\mu \mathrm{mol} / \mathrm{gdw})$. The quantities of individual SCFA ( $\mu \mathrm{mol} / \mathrm{gdw}$ of the enema samples), however, are not good indicators of differences in the fermentation patterns of the microbial communities. For example, differences in the liquid to solid ratio of the faecal material removed by the enema sample could lead to higher concentrations in one group versus another even if the fermentations were identical. A better indicator is given by the ratios of the individual major SCFA to total SCFA. For example, a higher ratio of propionate in one group reflects relatively greater activity of the bacteria in the microbial community that produce propionate.

The ratio comparisons of the subject groups are shown in Table 1. A highly significant difference in the SCFA ratios of the subjects who were found to have or had a history of tubular adenomatous (adenomatous), villotubular, villous adenomas, or colon cancer versus the normal group was found. The proportions of acetate were higher and the proportions of butyrate were lower for the polyp-cancer groups. There were no significant differences in the proportions of acetate, propionate, or butyrate between the diverticulosis groups versus the normal group.

The inflammatory bowel disease subjects had higher acetate and lower butyrate ratios than normal subjects but the ratios of the two groups were not significantly different. Most of the inflammatory bowel disease subjects had loose bowel movements or were taking sulphasalazine.

Because 15 subjects had diverticulosis and polyps and/or cancer, comparisons were made between polyp-cancer only and polyp-cancer including diverticulosis groups versus normal subjects, and diverticulosis only and diverticulosis including polypcancer groups versus normal subjects. The differences between the SCFA ratios of the polyp-cancer including diverticulosis and normal groups were essentially the same as the differences between the polyp-cancer only and the normal group except the proportion of isobutyrate in the polyp-cancer including diverticulosis group was significantly lower than in the normal group. There were no differences in the proportions of SCFA of the diverticulosis only or diverticulosis including polyp-cancer groups and the normal group. When the 11 cancer subjects incuding some with diverticula were considered as a discrete group, the acetate and butyrate ratios were significantly higher and lower, respectively, than in the normal group (Table 1).

Although the concentrations of individual SCFAgram dry weight per se are not good indicators of fermentation pattern differences, there were significant differences between groups. The concentration of total SCFA and the concentrations of acetate and

Table 2 Faecal SCFA concentrations in normal subjects and those with adenomatous polyps and/or diverticulosis

\begin{tabular}{|c|c|c|c|c|c|c|c|c|}
\hline \multirow[b]{2}{*}{ Diagnostic category } & \multicolumn{8}{|c|}{ umol/dry weight of enema* } \\
\hline & $n$ & Total SCFA & Acetate & Propionate & Isobutyrate & Butyrate & Isovalerate & Valerate \\
\hline Normal & 35 & $703(358)$ & $443(280)$ & $106(57)$ & $9(5)$ & $119(81)$ & $12(6)$ & $15(6)$ \\
\hline \multicolumn{9}{|l|}{ Polyp-cancer } \\
\hline No diverticula & 18 & $1146(508)[0 \cdot 0009]$ & $784(344)[0.0006]$ & $179(134)(0.007]$ & $11(6)$ & $137(75)$ & $15(7)$ & $20(10)[0.012]$ \\
\hline Including diverticula & 33 & $1001(559)[0 \cdot 01]$ & $677(401)[0.007]$ & $154(109)[0 \cdot 024]$ & $10(5)$ & $127(86)$ & $15(6)$ & $19(10)[0 \cdot 042]$ \\
\hline Colon cancer & 11 & $921(498)$ & $611(318)$ & $164(121)[0.03]$ & $8(5)$ & $109(79)$ & $12(4)$ & $17(12)$ \\
\hline \multicolumn{9}{|l|}{ Diverticula } \\
\hline No polyp-cancer & 39 & $953(625)[0.039]$ & $600(405)$ & $145(94)[0.034]$ & $16(25)$ & $148(100)$ & $20(33)$ & $24(23)[0.026]$ \\
\hline Including polyp-cancer & 54 & $918(612)$ & $586(411)$ & $139(86)[0.044]$ & $14(22)$ & $140(100)$ & $19(29)$ & $22(20)[0.045]$ \\
\hline Inflammatory bowel disease & 8 & $911(737)$ & $611(480)$ & $147(166)$ & $8(7)$ & $119(94)$ & $10(8 \cdot 8)$ & $16(18)$ \\
\hline
\end{tabular}

*Means (SD); p values comparing the means of diagnostic groups with the means of the normal group by a two tailed $t$ test are shown in square brackets where significant. 
propionate were significantly greater in the polypcancer group (Table 2). Of the minor SCFA, valerate was significantly higher for the same group of subjects. Subjects with diverticulosis had significantly higher concentrations of total SCFA and propionate than normal subjects, and a trend toward higher acetate and butyrate concentrations. Of the minor SCFA, there was a significantly higher concentration of valerate. As seen in Table 2, the differences between the polyp-cancer including diverticulosis group and normal subjects were less significant than for the polyp-cancer group alone. Acetate, valerate, propionate and total SCFA remained significantly higher, however, for the combined group. The relationships to the amounts of SCFA of the diverticulosis including polyp-cancer group to those of the normal group were essentially the same as for the diverticulosis only group (Table 2 ).

Differences in ratios and concentrations of SCFA were compared with respect to factors other than diagnostic groups. No significant correlations were found when the comparisons were made on the basis of age, subject weight or dry weights of samples using Pearson's product moment correlation. The only sex difference found was a higher proportion of isobutyrate for women $(1.7 \% \pm 1.5$ to total SCFA) versus men $(1.2 \% \pm 0.8$ of total SCFA) $(\mathrm{p}=0.04$ by a two tailed $t$ test).

\section{Discussion}

The significantly higher proportions of acetate and lower proportions of butyrate in samples of the polyp-cancer group probably reflect colonic microbial communities and fermentations that differ from those of the normal group. These results suggest an increase in the floral capacity to produce acetate and a decrease in the capacity to form butyrate in the polyp-cancer group. An alternative hypothesis to explain the different ratios is that the fermentations are the same in the polyp-cancer group and the normal group. This possibility seems unlikely, however, because all available evidence suggests that absorption of SCFA is a passive process without selective absorption. ${ }^{7}$ Another hypothesis would invoke the possibility of differences in the metabolism of the SCFA by the colonic cells of the polypcancer versus the normal group.

The significantly higher concentrations of total SCFA, acetate, propionate, and valerate per gram dry weight of the samples of the polyp-cancer group is difficult to explain. Possible explanations include a tendency for proportionally greater flushing of liquid than solids during the sampling of the polyp groupcancer group or greater liquid-to-dry-matter ratios of the faecal material removed by enema sampling.
Both possibilities would lead to a higher value of $\mu \mathrm{mol}$ SCFA/gram dry weight. The fact that a significant difference was not found for other acids such as butyrate may simply be because of the higher coefficient of variation of the respective determinations. For those SCFA that were not significantly different, the trend was toward higher mean concentrations for the polyp-cancer group.

We previously reported an increased incidence of high concentrations of methanogenic bacteria/gdw of enema samples in a diverticulosis group compared with normal subjects. $^{5}$ No difference was found between the polyp group and normal subjects. These results and those of the present study indicate there is no correlation between the presence of high concentrations of methanogens and the SCFA composition of the colonic contents of the different subject groups.

The possibility that the different SCFA ratios of the polyp-cancer group reflect decreased floral capacity to produce butyrate are particularly interesting in the context of reports that subjects with polyps malabsorb less starch than normal subjects ${ }^{8}$ and that the fermentation of starch tends to produce higher proportions of butyrate than the fermentation of glucose, xylan or pectin. ${ }^{29}$ This suggests that the colonic fermentation of starch and the production of butyrate would be greater in normal subjects because starch is more available for fermentation by the colonic microflora. As butyrate is a known regulator of cell differentiation, ${ }^{10}$ it is especially important to conduct additional experiments focused on possible relationships between starch malabsorption, production of butyrate by the colonic flora and the effect of butyrate concentrations in the colon on the development of polyps and colon cancer.

This study was supported in part by the Steven C. Clark Research Fund of The Mary Imogene Bassett Hospital and grant AI20244 from the National Institutes of Health. We thank $L$ Vadlamudi for technical assistance and Dr Thomas A Pearson for suggestions. Preliminary results of concentrations of short chain fatty acid analysis in normal subjects and subjects with diverticulosis has been published in abstract form. Gastroenterology 1985; 88: 1628.

\section{References}

1 Wolin MJ, Miller TL. Carbohydrate fermentation. In: Hentges DJ, ed. Human intestinal microflora in health and disease. New York: Academic Press, 1983: 147-65.

2 Weaver GA, Krause JA, Miller TL, Wolin MJ. Constancy of glucose and starch fermentations by two different human fecal microbial communities. Gut. In press. 
3 Bond JH, Engel RF, Levitt MD. Factors influencing pulmonary methane excretion in man. An indirect method of studying the in situ metabolism of the methane producing colonic bacteria. J Exp Med 1971; 133: 572-88.

4 Miller TL, Wolin MJ. Methanogens in human and animal intestinal tracts. System Appl Microbiol 1986; 7: 223-9.

5 Weaver GA, Krause JA, Miller TL, Wolin MJ. Incidence of methanogenic bacteria in a sigmoidoscopy population: an association of methanogenic bacteria and diverticulosis. Gut 1986; 27: 698-704.

6 Miller TL, Wolin MJ. Stability of Methanobrevibacter smithii populations in the microbial flora excreted from the human large bowel. Appl Environ Microbiol 1983; 45: $317-8$.

7 Cummings, JH. Colonic Absorption: the importance of short chain fatty acids in man. Scand $J$ Gastroenterol 1984; 93 suppl 19: 89-99.

8 Thornton JR. Dryden A, Kelleher J, Losowsky MS. Super-efficient starch absorption. A risk factor for colonic neoplasia. Dig Dis Sci 1987; 32: 1088-91.

9 Englyst HN, Hay S, McFarlane GT. Polysaccharide breakdown by mixed populations of human fecal bacteria. FEMS Microbiol Ecol 1987; 95: 163-71.

10 Kruh J. Effects of sodium butyrate, a new pharmacological agent, on cells in culture. Mol Cell Biochem 1982; 42: 65-82. 